\title{
Structural evolution of photocrosslinked silk fibroin and silk fibroin-based hybrid hydrogels: A small angle and ultra-small angle scattering investigation
}

Whittaker, Jasmin; Balu, Rajkamal; Knott, Robert; de Campo, Liliana; Mata, Jitendra; Rehm, Christine; Hill, Anita

https://researchrepository.rmit.edu.au/esploro/outputs/9921861714601341/filesAndLinks?institution=61RMIT_INST\&index=null

Whittaker, J., Balu, R., Knott, R., de Campo, L., Mata, J., Rehm, C., Hill, A., Dutta, N., \& Choudhury, N. (2018). Structural evolution of photocrosslinked silk fibroin and silk fibroin-based hybrid hydrogels: A small angle and ultra-small angle scattering investigation. International Journal of Biological Macromolecules, 114, 998-1007. https://doi.org/10.1016/j.ijbiomac.2018.03.044

Document Version: Accepted Manuscript

Published Version: https://doi.org/10.1016/j.ijbiomac.2018.03.044

Repository homepage: https://researchrepository.rmit.edu.au

CC BY-NC-ND V4.0

(C) 2018 Published by Elsevier B.V

Downloaded On 2023/04/26 21:08:11 +1000 


\title{
Structural evolution of photocrosslinked silk fibroin and silk fibroin-based hybrid hydrogels: A small angle and ultra-small angle scattering investigation
}

\author{
Jasmin L. Whittaker ${ }^{a}$, Rajkamal Balu ${ }^{b}$, Robert Knott ${ }^{c}$, Liliana de Campo ${ }^{\mathrm{c}}$, \\ Jitendra P. Mata ${ }^{c}$, Christine Rehm ${ }^{c}$, Anita J. Hill ${ }^{\text {d }}$, Naba K. Dutta a,b,*, Namita \\ Roy Choudhury a,b,*
}

${ }^{a}$ Future Industries Institute, University of South Australia, Mawson Lakes, SA 5095, Australia

${ }^{\mathrm{b}}$ School of Chemical Engineering, University of Adelaide, SA 5005, Australia ${ }^{\mathrm{c}}$ Australian Centre for Neutron Scattering, Australian Nuclear Science and Technology Organisation, Lucas Heights, NSW 2234, Australia

${ }^{\mathrm{d}}$ CSIRO Manufacturing, Bayview Avenue, Clayton, VIC 3168, Australia

*Corresponding Author Email: namita.roychoudhury@adelaide.edu.au;

naba.dutta@adelaide.edu.au 


\section{ABSTRACT}

Regenerated Bombyx mori silk fibroin (RSF) is a widely recognized protein for biomedical applications; however, their hierarchical gel structures are poorly understood. In this paper, the hierarchical structure of photocrosslinked RSF and RSF-based hybrid hydrogel systems: (i) $\mathrm{RSF} / \mathrm{Rec} 1-$ resilin and (ii) $\mathrm{RSF} /$ poly $(N$-vinylcaprolactam (PVCL) is reported for the first time using small-angle scattering (SAS) techniques. The structure of RSF in dilute to concentrated solution to fabricated hydrogels were characterized using small angle X-ray scattering (SAXS), small angle neutron scattering (SANS) and ultra-small angle neutron scattering (USANS) techniques. The RSF hydrogel exhibited three distinctive structural characteristics: (i) a Porod region in the length scale of 2 to $3 \mathrm{~nm}$ due to hydrophobic domains (containing $\beta$-sheets) which exhibits sharp interfaces with the amorphous matrix of the hydrogel and the solvent, (ii) a Guinier region in the length scale of 4 to $20 \mathrm{~nm}$ due to hydrophilic domains (containing turns and random coil), and (iii) a Porod-like region in the length scale of few micrometers due to water pores/channels exhibiting fractal-like characteristics. Addition of Rec1-resilin or PVCL to RSF and subsequent crosslinking systematically increased the nanoscale size of hydrophobic and hydrophilic domains, whereas decreased the homogeneity of pore size distribution in the microscale. The presented results have implications on the fundamental understanding of the structure-property relationship of RSF-based hydrogels.

Keywords: Silk fibroin, Rec1-resilin, poly(N-vinylcaprolactam), hybrid hydrogel, smallangle scattering, hierarchical structure 


\section{Introduction}

In recent years, substantial research interest has been focused on the development of novel peptide- and protein-based materials for biomedical application [1-3]. In particular, silk fibroin protein has been recognized as a very promising material for hydrogel-based scaffolds due to its excellent biocompatibility, high mechanical strength, abundance in nature and biodegradability [4-6]. Hydrogels are crosslinked, hydrated polymer networks widely applied in the field of tissue engineering and regenerative medicine [7]. Aqueous and organic solutions of silk fibroin can be obtained from cocoons of silk worms (e.g. Bombyx mori) through chemical regeneration methods, and the regenerated silk fibroin (RSF) can be fabricated into hydrogels through various processing techniques inducing physical and/or chemical crosslinking between RSF [8, 9]. The RSF-based hydrogels have been reported to show a great variety of biomedical applications [10]. In our previous work, a facile fabrication of threedimensional (3D) Bombyx mori RSF hydrogels was presented using a ruthenium catalyst mediated photocrosslinking method. The fabricated hydrogels exhibited good biocompatibility and elastic modulus in the mega Pascal (MPa) scale [11]. Despite fast crosslinking time and advantageous properties, there remained a need to tune the swelling capability and mechanical properties of the hydrogels for targeted tissue engineering applications. The mechanical requirements for such applications vary greatly depending on the location, function and characteristics of the human tissue; such as for articular cartilage tissue engineering a modulus of $\sim 500$ to $1000 \mathrm{kPa}$ is require [12]. Therefore, to achieve the aforesaid goal of tuning the physicochemical properties of photocrosslinked RSF hydrogels, an initial investigation into the co-assembly behaviour of RSF with Rec1-resilin and/or poly( $N$-vinylcaprolactam) (PVCL) was performed [13]. Rec1-resilin is a biomimetic multi-stimuli responsive elastomeric insect protein, which upon crosslinking demonstrates resilience (recovery after deformation) exceeding that of unfilled synthetic polybutadiene rubber [14]. PVCL is a synthetic thermo- 
responsive and biocompatible polymer widely applied in the biomedical field [15]. Combining the rigid phase of RSF with softer and/or thermo-responsive phase of Rec1-resilin and/or PVCL, the ability of RSF to tune the phase transition behaviour of Rec1-resilin and/or PVCL was demonstrated [13]. Subsequently, RSF and Rec1-resilin proteins were chemically cocrosslinked to form a hybrid hydrogel via the established photocrosslinking method [16]. The fabrication of RSF/PVCL double network (DN) hybrid hydrogel with the PVCL physically entrapped into the photocrosslinked RSF phase was also demonstrated recently [17]. The detailed characterisation including water uptake, molecular chain dynamics, in vitro cytocompatability and mechanical properties of the fabricated hybrid hydrogels are presented elsewhere $[16,17]$.

The molecular weight distribution and crystallinity of RSF is commonly affected by the chemicals used for regeneration [18-20]. Although regeneration of silk fibroin using lithium bromide is quite popular, RSF preparation using calcium salts is cost effective and environmentally benign [21]. Moreover, RSF prepared using calcium chloride and ethanol has been reported for relatively higher molecular weight (compared to methods using calcium nitrate and ethanol/methanol) and silk I structure (hydrated phase), which is of interest for hydrogel applications and therefore used in this work $[19,20]$. Unlike physical gelation in silk caused by $\beta$-sheet formation, the photo-chemical crosslinking of RSF and/or Rec1-resilin solutions involves the formation of covalent di-tyrosine crosslinks between tyrosine amino acid residues of protein chains $[11,22]$. In the case of co-crosslinked RSF/Rec1-resilin hydrogel, the di-tyrosine crosslinks may occur between RSF-Rec1-resilin, RSF-RSF and Rec1-resilinRec1-resilin chains [16]. However, in the case of the RSF/PVCL DN hydrogel, only the RSF chains are chemically crosslinked and the PVCL is physically entrapped in the network forming hydrogen bonds with RSF [17]. Although formation of di-tyrosine is the main mode of crosslinking in the ruthenium-mediated photocrosslinking method a number of other physical 
interactions and/or conformational changes may also contribute to the self-assembly and microstructure formation in RSF-based hydrogels [23]. Therefore, it can be reasonably assumed that in RSF-based hybrid hydrogel systems multiple interactions may occur and contribute to the changes in microstructure of hydrogels that in turn results in tuning of their final material properties.

In order to develop RSF-based functional hybrid hydrogels with tuneable physicochemical properties an in-depth understanding of their hierarchical structure, its role on the bulk properties and how the molecular building blocks transmit properties across the lengthscales in the macroscopic sample is crucial. The molecular structure and morphology of RSF and their self-assembled aggregates (formed via physical interaction) have been reported in literature using small-angle scattering (SAS), atomic force microscopy (AFM) and scanning electron microscopy (SEM) [24-27]. The structure of physically crosslinked (self assembled) RSF hydrogel has been reported to consist of fibroin proteins which form thin strands by $\beta$ sheet formation below a length scale of $15 \mathrm{~nm}$ and aggregate randomly to create the self similar structures of higher length scales, which randomly aggregate to form micron size protein-rich particles that form connected network $[22,24]$. However, the structure of chemically crosslinked RSF hydrogel is not fully understood. The knowledge of the hierarchical structure (nano- to micro-scale) of chemically crosslinked RSF hydrogels is critical to understand their structure-property relationship and physicochemical differences against physically crosslinked hydrogels. Moreover, to our knowledge there is no report that discusses the structure of RSFbased hybrid hydrogel systems, particularly RSF/Rec1-resilin and RSF/PVCL systems, which is essential to understand their structure related property enhancements. Small-angle X-ray scattering (SAXS) is an established SAS method for low-resolution structural characterization of biological macromolecules in dilute solutions, and is complementary to the high resolution X-ray crystallography and nuclear magnetic resonance techniques [28, 29]. On the other hand, 
small-angle neutron scattering (SANS) is a powerful technique for investigating the nanostructure and organization of protein polymers in concentrated solutions and hydrogels, and is complementary to SAXS with high sensitivity to lighter elements such as hydrogen [30, 31]. The structural characterization of hydrogels up to a length scale of several micrometers can also be studied using ultra-small-angle neutron scattering (USANS) [32]. Therefore, combining SAXS, SANS and USANS, we investigate and report the structural evolution of photocrosslinked pristine RSF and RSF-based hybrid hydrogels for the first time.

\section{Materials and methods}

\subsection{Materials}

Bombyx mori silk fibers were purchased from Beautiful Silks, Australia. Deuterium oxide $\left(\mathrm{D}_{2} \mathrm{O}\right)$ was kindly supplied by the Australian Nuclear Science and Technology Organisation (ANSTO). All other chemicals used in this study were of analytical grade purchased from Sigma-Aldrich, Australia. Aqueous solution of silk fibroin was prepared via chemical regeneration method using calcium chloride [11]. Rec1-resilin was synthesized via recombinant DNA technology using exon 1 of the Drosophila Melanogaster gene CG15920 [33]. Poly( $N$-vinylcaprolactam) (PVCL) was prepared via free radical polymerization of the vinyl caprolactam monomer using 2,2'-azoisobutyronitrile [17]. The molecular weight of synthesized RSF, Rec1-resilin and PVCL were measured to be 37 to $200 \mathrm{kDa}, \sim 28.5 \mathrm{kDa}$ and $\sim 35.4 \mathrm{kDa}$, respectively $[16,17,29]$. The details of synthesis and molecular weight determination of the macromolecules are provided in the Supplementary Information.

\subsection{Preparation of hydrogels}

The hydrogels were prepared via a rapid ruthenium-mediated photocrosslinking method described in our previous work $[11,16,17]$. Briefly, the photo-catalyst Tris(2,2'bipyridyl)dichlororuthenium(II) hexahydrate $\left(\mathrm{Ru}(\mathrm{bpy})_{3}\right)$ and the electron acceptor ammonium 
persulfate (APS) were mixed with RSF solution (preventing exposure to light) using a magnetic stirrer. The solution mixture was then poured into Teflon moulds $(30 \mathrm{~mm}$ diameter and $1 \mathrm{~mm}$ depth) and exposed to white light $(250 \mathrm{~W})$ for 2 minutes. The gels formed were then turned over and exposed for additional 30 seconds to ensure complete crosslinking. For RSF-based hybrid hydrogel preparation, predefined amount of Rec1-resilin or PVCL was first mixed with RSF in volumetric ratios before adding $\mathrm{Ru}(\mathrm{bpy})_{3}$ and APS. In all cases the final solution concentration of $\mathrm{Ru}(\mathrm{bpy})_{3}$ and APS was kept at $5 \mathrm{mM}$ and $28 \mathrm{mM}$, respectively. The composition and codes of fabricated hydrogels are given in Table 1. The excess reactants from crosslinking reaction were removed from fabricated hydrogels by dialysis against MilliQ water $\left(\mathrm{H}_{2} \mathrm{O}\right)$. The dialysed samples were air-dried at ambient temperature followed by vacuum drying.

Table 1. Hydrogel sample composition and codes.

\begin{tabular}{lcc}
\hline Samples & \multicolumn{2}{c}{ Weight ratio } \\
\cline { 2 - 3 } & RSF:Resilin & RSF:PVCL \\
\hline RSF100 gel & $1: 0$ & $1: 0$ \\
RSF50 Resilin50 gel & $0.75: 1$ & - \\
RSF90 PVCL10 gel & - & $27.0: 1$ \\
RSF80 PVCL20 gel & - & $12.0: 1$ \\
RSF70 PVCL30 gel & - & $7.0: 1$ \\
RSF60 PVCL40 gel & - & $4.5: 1$ \\
\hline
\end{tabular}

\subsection{Small-angle X-ray scattering (SAXS)}

SAXS experiments were performed using a NanoSTAR II SAXS instrument (Bruker AXS, Karlsruhe Germany). The solution samples (prepared in $\mathrm{H}_{2} \mathrm{O}$ ) were loaded into $2 \mathrm{~mm}$ ID quartz capillaries and the temperature was maintained at $25^{\circ} \mathrm{C}$. The scattering intensity, $\mathrm{I}(q)$ of samples were recorded against the scattering vector, $q$ (equation 1 ) in the range $0.01 \AA^{-1}$ to 0.39 $\AA^{-1}$ [34]. 


$$
q=\frac{4 \pi \sin \theta}{\lambda}
$$

where $2 \theta$ is the angle of scattering and $\lambda$ is the wavelength of the X-ray beam $(1.54 \AA)$. The obtained $\mathrm{I}(q)$ was radially averaged using routines in the Bruker software. The appropriate buffer and instrument background was subtracted using the PRIMUS computer program [35].

\subsection{Small-angle neutron scattering (SANS)}

SANS experiments were performed using the Quokka SANS instrument at ANSTO [36]. Source to sample aperture distances of 2,12 and $20 \mathrm{~m}$ with neutron wavelength of 5 and 8.1 $\AA^{-1}$, respectively were used to measure $\mathrm{I}(q)$ over a $q$-range of $0.0007 \AA^{-1}$ to $0.35 \AA^{-1}$. A sample aperture diameter of $12.5 \mathrm{~mm}$ was used for all three configurations. The scattering of samples were measured at a fixed temperature of $25^{\circ} \mathrm{C}$. The solution samples $\left(15 \mathrm{wt} \%\right.$ prepared in $\mathrm{H}_{2} \mathrm{O}$ and $2 \mathrm{wt} \%$ prepared by dialysis against $\mathrm{D}_{2} \mathrm{O}$ ) were loaded in Hellma cells with $20 \mathrm{~mm}$ diameter and $1 \mathrm{~mm}$ path length for analysis. The dehydrated gels were first soaked in $\mathrm{D}_{2} \mathrm{O}$ to obtain a constant swollen weight. The $\mathrm{D}_{2} \mathrm{O}$ equilibrium swollen hydrogels were cut into discs (ca. 19 $\mathrm{mm}$ diameter) and loaded into demountable Quokka cells of $20 \mathrm{~mm}$ diameter and $1 \mathrm{~mm}$ path length along with excess $\mathrm{D}_{2} \mathrm{O}$. The neutron scattering length density (SLD) of RSF $\left(\sim 3.8 \times 10^{-}\right.$ $\left.{ }^{6} \AA^{-2}\right)$ [37], Rec1-resilin $\left(\sim 2.8 \times 10^{-6} \AA^{-2}\right)$ [31] and PVCL $\left(\sim 1.2 \times 10^{-6} \AA^{-2}\right)$ [38] provides good contrast against $\mathrm{D}_{2} \mathrm{O}\left(6.36 \times 10^{-6} \AA^{-2}\right)$, and also reduce any incoherent background scattering from hydrogen in the system. SANS data were reduced using NCNR SANS reduction macros (modified for the QUOKKA instrument) using the Igor software package with data corrected for empty cell scattering and transmission. Data collected were transformed to absolute scale using an attenuated direct beam transmission measurement [39]. The appropriate buffer and instrument background was subtracted using the PRIMUS computer program [35].

\subsection{Ultra-small angle neutron scattering (USANS)}


USANS experiments were performed using the Kookaburra USANS instrument at ANSTO using neutron wavelength of $4.74 \AA[40]$. The $\mathrm{D}_{2} \mathrm{O}$ equilibrium swollen hydrogel samples were cut into discs $(25 \mathrm{~mm} \times 25 \mathrm{~mm})$ and loaded into demountable Kookaburra cells of $30 \mathrm{~mm} \times 30$ $\mathrm{mm}$ (length $\times$ height) and $1 \mathrm{~mm}$ path length along with excess $\mathrm{D}_{2} \mathrm{O}$. All the measurements were conducted at room temperature with a sample aperture diameter of $20 \mathrm{~mm}$. Data were collected in the $q$-range of $3 \times 10^{-5} \AA^{-1}$ to $1.8 \times 10^{-2} \AA^{-1}$. Data collected for samples were reduced with an empty Kookaburra cell data (background) using Python scripts running in Gumtree based on the standard procedure [41]. The experimental data were de-smeared using the Lake algorithm incorporated in the NIST USANS macros, and subsequently merged with the SANS data for analysis [39]. The incoherent background scattering from samples determined with a high- $q$ power law fit $\left(0.15 \AA^{-1}<\mathrm{q}<0.3 \AA^{-1}\right)$ using the SasView computer program was subtracted from the respective data for analysis [42]. The structural parameters of the samples were determined by fitting the scattering data with appropriate models and/or functions using the SasView computer program (http://www.sasview.org/).

\section{Results and discussion}

\subsection{Intrinsic structure of synthesized macromolecules in dilute solution}

Fig. 1A shows the SANS curves $\left(0.01<q<0.35 \AA^{-1}\right)$ of synthesized PVCL, Rec1-resilin and RSF solutions at $2 \mathrm{wt} \%$ concentration. The secondary structure of RSF and Rec1-resilin has been reported to be largely unordered (random coil) using circular dichroism spectroscopy [13, 29, 43]. In SAS, the unfoldedness or random coil likeliness of macromolecules can be qualitatively assessed by means of the Kratky plot $\left(\ln \mathrm{I}(q)^{*} q^{2}\right.$ vs. $q$ ), where the plot divides-out the decay of the scattering and show certain other features more evident [44]. Kratky plots (Fig. 1B) of all samples displayed initial monotonic increase in the low- $q$ region followed by a plateau or slight increase in high- $q$, which is characteristic of an overall unfolded conformation of proteins and polymers in dilute solutions [45]. The SANS curves of samples demonstrated 
two distinctive regions: a high- $q$ Porod region $\left(0.025<q<0.35 \AA^{-1}\right)$ and a mid- $q$ Guinier region $\left(0.007<q<0.025 \AA^{-1}\right)[13]$. The Porod exponent or slope that can be determined from Porod region (high- $q$ ) provides information about the "fractal dimension" of the scattering objects, whereas the Guinier region (low- $q$ ) allows for the estimation of the radius-of-gyration $\left(R_{g}\right)$ of the scattering object [44]. Therefore, the scattering data in the Porod region $(0.05<q<$ $0.35 \AA^{-1}$ ) were fit with a Power law function (Fig. 1C) and the estimated structural parameters are given in Table 2. All samples exhibited Porod slope between $\sim 1.7$ (signature of fully swollen polymer coils) and 2.0 (signature of Gaussian chains in dilute environment) [46]. Therefore, the intrinsic molecular structure of synthesized macromolecules is established to be predominantly unordered or random coil.
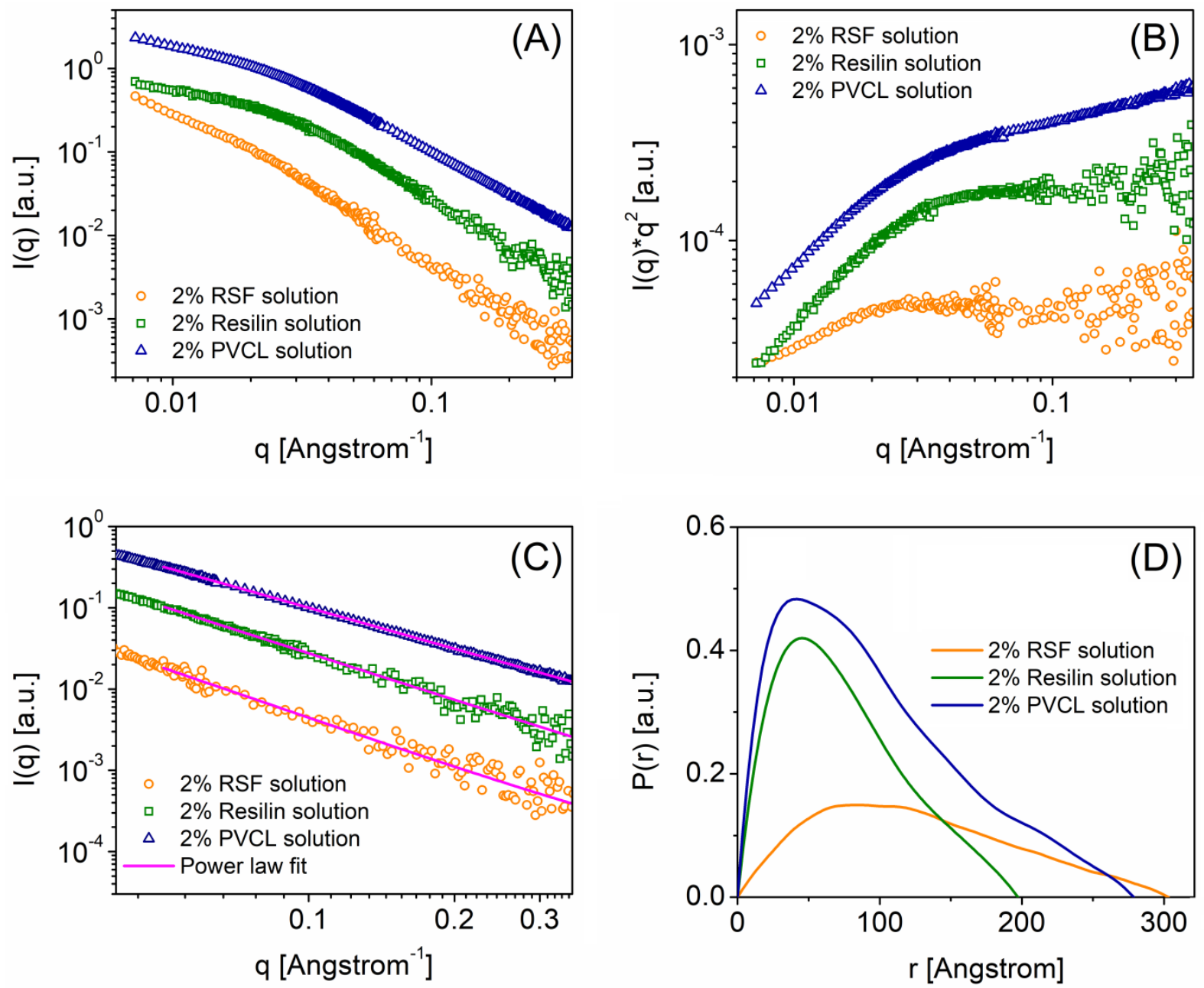
Fig. 1. (A) SANS curve, (B) Kratky plot, (C) Porod region power law fit and (D) P(r) distribution function fit of synthesized Rec1-resilin, PVCL and RSF, respectively.

Table 2. Structural parameters of solution samples determined from SANS data.

\begin{tabular}{lcc}
\hline Samples & Power law fit & $\begin{array}{c}\text { P(r) function } \\
\text { fit }\end{array}$ \\
\cline { 2 - 3 } & Porod slope & Rg (nm) \\
\hline $2 \mathrm{wt} \%$ Resilin & $1.93 \pm 0.02$ & $6.04 \pm 0.06$ \\
$2 \mathrm{wt} \%$ PVCL & $1.70 \pm 0.01$ & $7.97 \pm 0.01$ \\
$2 \mathrm{wt} \%$ RSF & $2.00 \pm 0.01$ & $11.01 \pm 0.34$ \\
\hline
\end{tabular}

In polymer physics, the term radius of gyration $\left(R_{g}\right)$ is commonly used to describe the size of macromolecular configuration in solutions. In SAS, Guinier approximation is widely applied to estimate the $R_{g}$ of scattering object [46]. However, Guinier approximation is very sensitive to scattering intensities at small $q$-values and has been reported to underestimate the $R_{g}$ for structurally unordered protein and polymer coils [47]. On the other hand, RSF prepared using calcium chloride and ethanol has been reported for broad molecular weight distribution using gel permeation chromatography [48]. Therefore, RSF prepared in this work is likely to be polydispersed. With the intrinsic structure of synthesized macromolecules established to be predominantly unordered using Kratky plot and Porod slope, their $R_{g}$ was determined using the pair-distance distribution function, $\mathrm{P}(\mathrm{r}) . \mathrm{P}(\mathrm{r})$ is a model independent function and calculates the $R_{g}$ by inverse Fourier transform of the entire scattering spectrum with a histogram of all of the inter-atomic distances (r) [44, 49]. The obtained $R_{g}$ of $11.01 \pm 0.34 \mathrm{~nm}$ for RSF, $6.04 \pm 0.06$ $\mathrm{nm}$ for Rec1-resilin and $7.97 \pm 0.01 \AA$ for PVCL from $\mathrm{P}(\mathrm{r})$ function fit (using the PRIMUS computer program) are generally in good agreement with values reported previously [24, 31 , 50]. Moreover, the shape of the $\mathrm{P}(\mathrm{r})$ distribution curves with asymmetric and extended tail region (Fig. 1D) suggest the synthesized macromolecules to be elongated in dilute solutions [44]. 


\subsection{Pre-gelation structure of RSF in concentrated solution}

Increase in concentration of proteins in aqueous solutions reduce their available volume and elevate the osmotic pressure relative to pure water, thereby causing them to adapt to more compact conformations than those in dilute condition. This effect is commonly referred to as "osmotic stress" [51]. Therefore, to study the supramolecular structural organization of protein domains in hydrogels (commonly formed by crosslinking of protein macromolecules in concentrated solutions) it is crucial to understand the molecular structure and organization of protein macromolecules from dilute to concentrated solutions. The RSF macromolecules contains both hydrophilic and hydrophobic amino acid segments and is reported to form nanostructures in neutral solutions due to repulsive force balance between interior and exterior negative charge [52]. At very low concentrations $(<0.003 \%)$, it is reported that the molecular structure of RSF can be seen as nanofibrils by AFM. However, upon increase in concentration the repulsive force balance between interior and exterior negative charge is affected (without any secondary structural change), thereby leading to formation of aggregates (nanoparticles and protofibrils) $[26,52]$. 

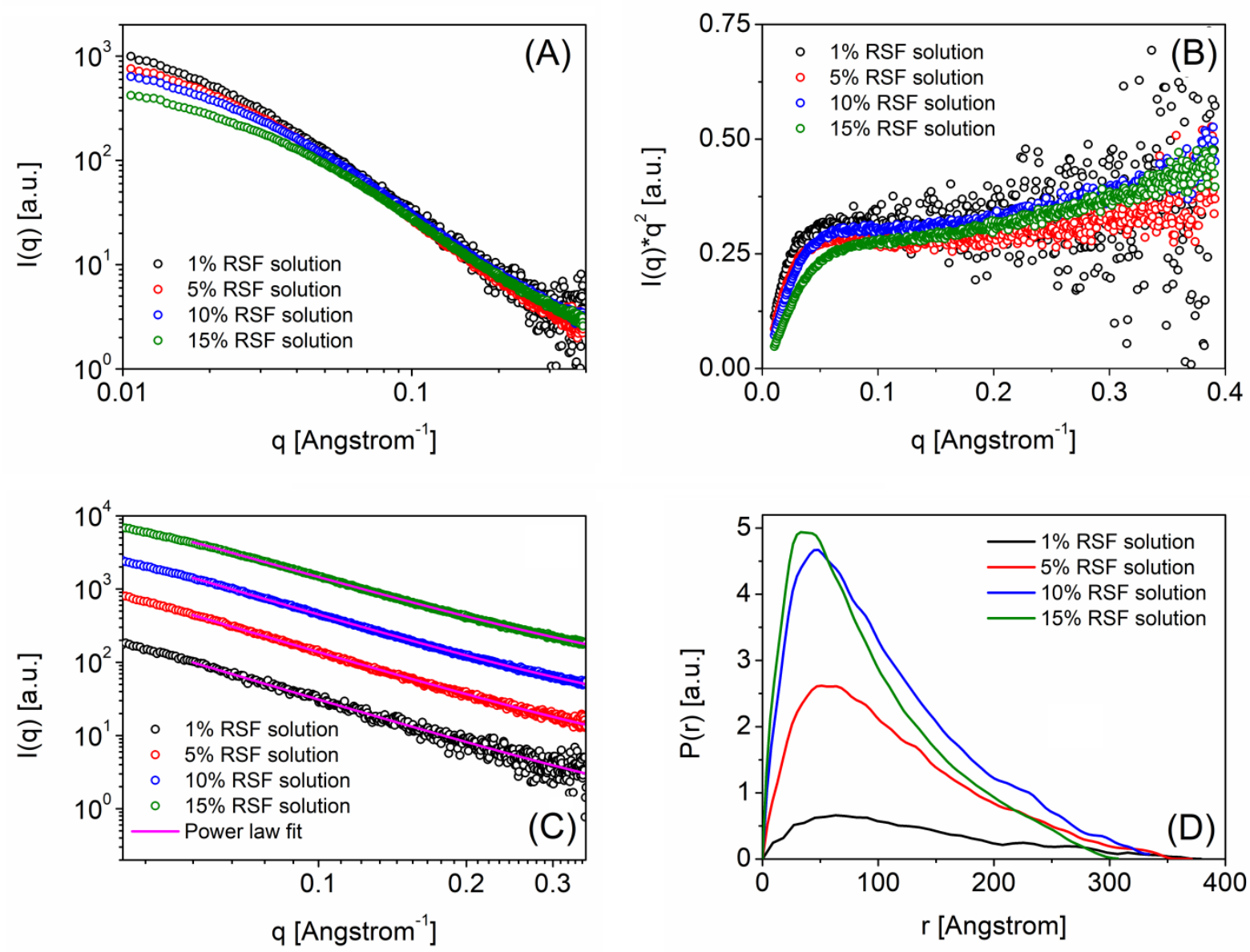

Fig. 2. (A) SAXS curve (normalized), (B) Kratky plot (normalized), (C) Porod region power law fit and (D) P(r) function fit of RSF as a function of solution concentration.

From our previous experiments, a minimum RSF concentration of $10 \mathrm{wt} \%$ was determined for successful fabrication of hydrogel by ruthenium mediated photocrosslinking method [53]. The RSF hydrogel in this study was fabricated at $15 \mathrm{wt} \%$ concentration. Therefore, the intrinsic structure and organization of RSF in 1 to $15 \mathrm{wt} \%$ solution was studied using SAXS. Fig. 2A shows the SAXS curves $\left(0.01<q<0.39 \AA^{-1}\right)$ of RSF (normalized to volume fraction) as a function of solution concentration. The Kratky plots showed an initial monotonic increase in low- $q$ region followed by a plateau or slight increase in high- $q$ (Fig. 2B), which is characteristic of an overall unfolded conformation of protein in solutions, and observed to be consistent with SANS results [45]. Porod slope and $R_{g}$ were subsequently estimated using power law fit $\left(0.05<q<0.35 \AA^{-1}\right)$ (Fig. 2C) and $\mathrm{P}(\mathrm{r})$ function fit, respectively. 
The estimated structural parameters are given in Table 3. The $1 \mathrm{wt} \%$ RSF solution exhibited a Porod slope of $\sim 2.0$, which is signature of Gaussian chain in dilute environment and is consistent with SANS results [46]. However, with increase in concentration the RSF solution showed decrease in low- $q$ intensity along with slight reduction in Porod slope. The $R_{g}$ was also observed to show a decreased trend with increase in concentration. This suggests an increased external repulsive interaction between RSF molecules with increase in concentration causing the protein chain to adapt to swollen coil conformation $[54,55]$. The observed Porod slope of $\sim 1.7$ (characteristics of swollen coil in good solvent) for $15 \mathrm{wt} \%$ RSF solution establishes the intrinsic molecular structure to be predominantly random coil from dilute to concentrated solution. Since the scattering intensity showed an increased trend towards low- $q$ in general, and the results may have been limited by the SAXS instrument $q$-range the molecular structure and organization of RSF at crosslinking concentration (15 wt \%) was further analysed using SANS with an extended low- $q$ measurement.

Table 3. Structural parameters of RSF as a function of solution concentration determined from SAXS data.

\begin{tabular}{lcc}
\hline Concentration (wt\%) & Power law fit & P(r) function fit \\
\cline { 2 - 3 } & Porod slope & $\mathbf{R}_{\mathbf{g}}(\mathbf{n m})$ \\
\hline 1 & $2.00 \pm 0.01$ & $10.65 \pm 0.35$ \\
5 & $1.89 \pm 0.01$ & $9.16 \pm 0.27$ \\
10 & $1.81 \pm 0.01$ & $8.44 \pm 0.14$ \\
15 & $1.71 \pm 0.01$ & $7.65 \pm 0.08$
\end{tabular}

Fig. 3A shows the SANS curve of $15 \mathrm{wt} \%$ RSF solution. The plot shows three distinctive scattering regions: low- $q\left(0.001<q<0.007 \AA^{-1}\right)$, mid- $q\left(0.007<q<0.025 \AA^{-1}\right)$ and high- $q\left(0.025<q<0.4 \AA^{-1}\right)$. The $15 \mathrm{wt} \%$ RSF solution exhibited scattering trend similar to that of $2 \mathrm{wt} \%$ solution (Fig. 1A) at high- $q$. However, between mid- $q$ to low- $q$ a clear Guinier region and an upturn (Porod-like region) were observed, which signifies intermolecular 
interactions and aggregation in the system. Similar behaviour has also been previously reported for concentrated RSF solutions prepared using lithium bromide [25]. On the other hand, USANS (very low- $q$ ) was employed to examine whether any large (microscale) structures are present in the solution and to provide a basis for comparison with the crosslinked RSF hydrogel. However, no significant scattering was observed above the background. The fractal dimension of RSF at high- $q$ was estimated using a Power law fit $\left(0.05<q<0.35 \AA^{-1}\right.$, Fig. $\left.3 \mathrm{~A}\right)$. An estimated Porod slope of $1.70 \pm 0.02$ illustrates the overall equilibrium swollen coil nature of the RSF, which is in good agreement with SAXS results [46]. The Kratky plot (Fig. 3B) showed an initial monotonic increase in the low- $q$ region followed by a plateau and a second monotonic increase in the high- $q$ region followed by a second plateau or slight increase. This behaviour emphasizes the presence of two structural domains in the system. It is reported that RSF (prepared using lithium bromide) at relatively dilute concentration ( $1 \mathrm{wt} \%)$ self-assemble to form micelles $(20-200 \mathrm{~nm})$ in aqueous solution by a thermodynamically driven process, whereas at high concentrations $(20 \mathrm{wt} \%)$ the micelles self-assemble into nanofibrils to reduce repulsive force [27]. However, in this study, the synthesized RSF between 1 to $15 \mathrm{wt} \%$ concentrations did not show micellar and/or fibrillar characteristic scattering profiles, which may be attributed to polydispersity in the system [56, 57]. Further, we have tried to estimate the $R_{g}$ of synthesized RSF and the fractal dimension of their aggregates at $15 \mathrm{wt} \%$ concentration. The SANS data was fit (Fig. 3C) with a shape-independent form factor function with polyGaussian coil model, $\mathrm{A}(q)$ at high- $q$ and a power law function, $\mathrm{B}(q)$ at low- $q$ as given in equation 2.

$$
I(q)=A(q)+B(q)
$$

The fit yielded a high- $q R_{g}$ of $7.51 \pm 0.06 \mathrm{~nm}$ and the fractal dimension of their aggregates as $2.04 \pm 0.03$. The obtained $R_{g}$ is in general agreement with SAXS results and the fractal dimension suggests loose aggregate structures. 

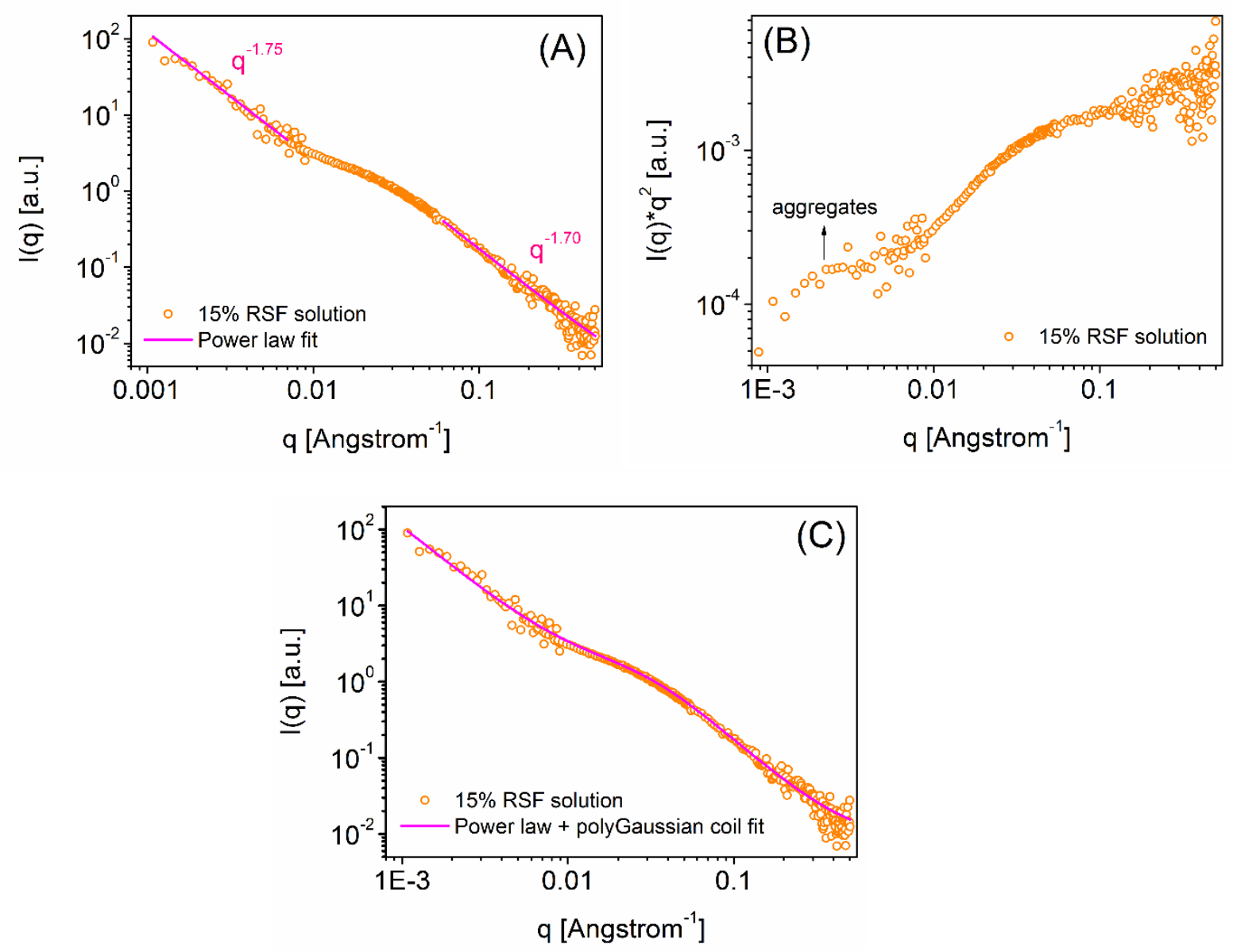

Fig. 3. (A) SANS curve with power law fits (Porod regions), (B) Kratky plot and (C) SANS curve with combined power law and polyGaussian coil fit of $15 \mathrm{wt} \%$ RSF solution.

\subsection{Supramolecular structure of photocrosslinked RSF hydrogel}

Fig. 4A shows the scanning electron microscopic image (cross-section) of freeze dried photocrosslinked RSF hydrogel. The hydrogel exhibited irregular polygonal or collapsed honeycomb-like 3D structures with interconnected pores similar to ones reported for freeze dried solutions of RSF and gamma-ray crosslinked RSF hydrogels [24, 58]. An average pore size of $2.29 \pm 0.77 \mu \mathrm{m}$ was estimated from the SEM image. However, visibility of any nanostructural feature was limited by the SEM instrument. The nano- to microstructure of RSF hydrogel was studied in detail using SANS and USANS. The SANS data of RSF hydrogel provided information about the frozen-in crosslinked network structure at the nanoscale, whereas the USANS region provided information about microscale structural inhomogeneities 
such as micropores that act as reservoirs and channels for water $[59,60]$. The SANS curve of RSF hydrogel (Fig. 4B) demonstrated a high- $q$ Porod region $\left(0.1<q<0.3 \AA^{-1}\right)$, a mid- $q$ to low- $q$ Guinier region $\left(0.005<q<0.05 \AA^{-1}\right)$ and a very low- $q$ Porod-like region $(0.0002<q<$ $\left.0.002 \AA^{-1}\right)$. A high- $q$ Porod slope of $4.0 \pm 0.01$ estimated using power law fit indicates formation of nanoscale hydrophobic domains/water poor regions upon photocrosslinking that exhibit sharp interface with the surrounding environment (amorphous hydrogel matrix and solvent) [44]. The Guinier region with increased scattering intensity suggests presence of secondary domain (presumably hydrophilic) in the nanoscale range, which contributes to the overall intensity. Silk fibroin is a semicrystalline material with the primary structure mainly consisting of the hexa amino acid sequences of GAGAGS and GAGAGY. The repeating polypeptides are held together by hydrogen bonds forming the $\beta$-sheet crystals (silk II conformation) that contribute to silk's rigid structure and tensile strength [61]. Apart from highly regular crystalline segments there are also several other types of irregular sequences including N-terminus, C-terminus, light chain, GT GT, GY GY and GAAS that contributes to the silk's amorphous structure (silk I conformation) and water uptake properties $[62,63]$. The $B$. mori silk fibroin contains $\sim 5 \mathrm{~mol} \%$ of tyrosine (Y) in its overall structure and is located in regular and irregular sequences. The presence of tyrosine in regular sequences of protein favours $\beta$-sheet crystals and irregular sequences of protein favours random coil [62, 64-66]. The soluble form of silk fibroin is in $\alpha$-helix and random coil conformations and by transitioning to $\beta$-sheets silk is rendered insoluble [67]. Recently, Drnovsek et. al. [58] presented the nanostructure of freeze dried RSF scaffolds (using transmission electron microscopy, TEM) to contain $\beta$-sheet crystallites ( $\sim 10$ strands) distributed in the amorphous matrix of silk fibroin. In photocrosslinked RSF hydrogel, the silk fibroin chains are connected by di-tyrosine crosslinks with a $\beta$-sheet, strands, turn and random coil content estimated to be $\sim 13 \%, 34 \%, 35 \%$ and $18 \%$, respectively (presented in our previous publication) [11]. 
Therefore, it is reasonable to assume the structure of photocrosslinked RSF hydrogel to contain hydrophobic domains/water poor regions (dominated by $\beta$-sheets) and hydrophilic domains/water rich regions (dominated by turns and random coil) in the nanoscale. The USANS region (very low- $q$ ) show strong upturn with a power law behaviour of $\sim q^{-3}$, typically observed for mass fractals suggesting structural inhomogeneities on a range of length scales in the sample extending beyond the USANS range [68]. On top of this steep upturn there is a broad feature, which is likely to stem from the micropores observed in the SEM image of the hydrogel.
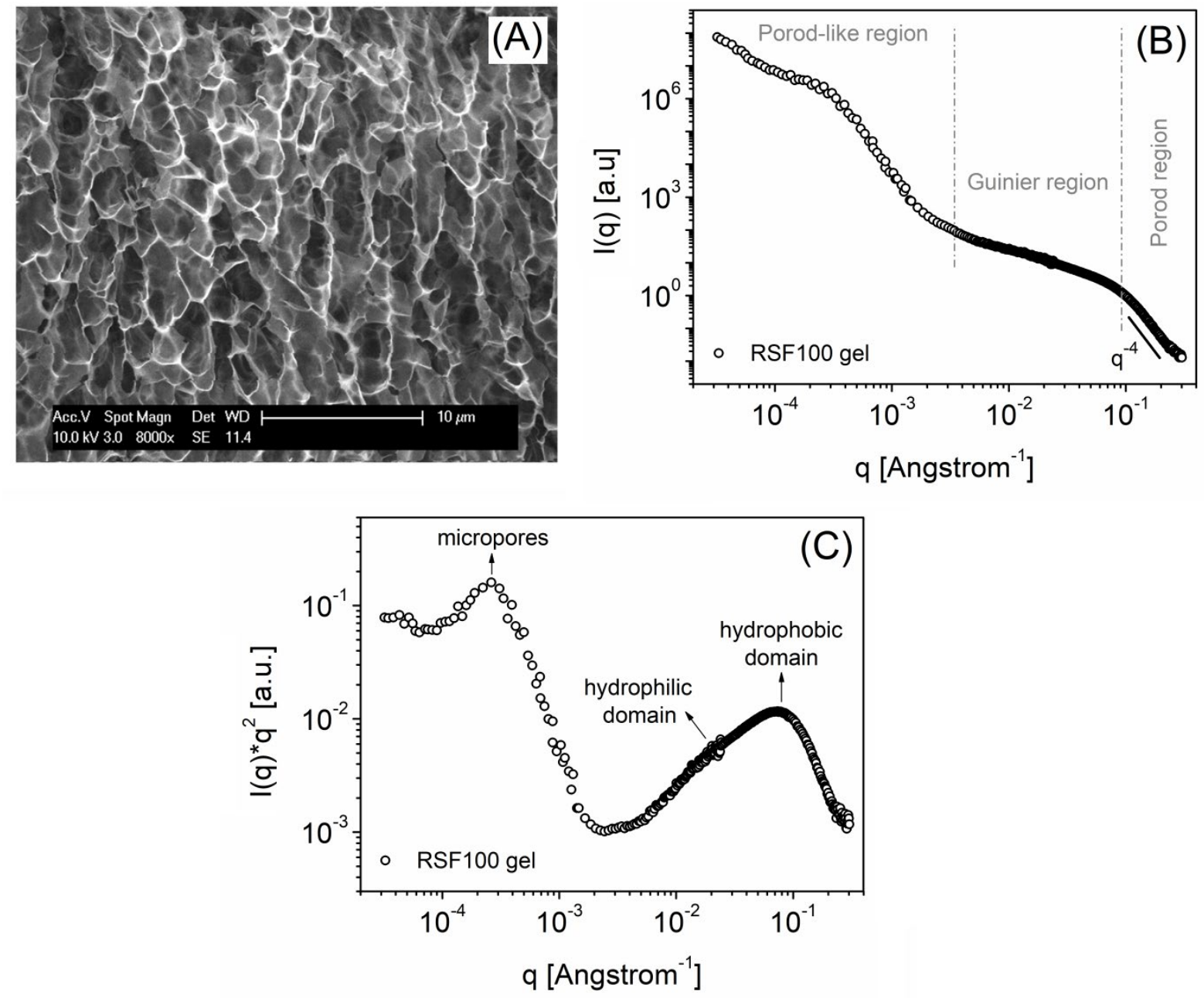

Fig. 4. (A) SEM image, (B) combined SANS and USANS curve and (C) Kratky plot of photocrosslinked RSF hydrogel. 
The Kratky plot of RSF hydrogel (Fig. 4C) clearly showed two peaks in USANS and SANS regions, respectively. The USANS correlation peak observed around $q$ value of 0.00025 $\AA^{-1}$ corresponds to a domain size (d) of $\sim 2.5 \mu \mathrm{m}$ (calculated using the relation $\mathrm{d}=2 \mathrm{Pi} / q$ ), which is in good agreement with average pore size measured from the SEM results [69]. The asymmetric peak observed with a broad shoulder in SANS region supports the presence of two nanoscale domains or phases in the system $[46,70]$. The structural parameters of the RSF hydrogel was estimated using a shape-independent form factor function with Guinier-Porod model, $\mathrm{A}(q)$ at high- $q$, Debye Anderson Brumberger (DAB) model, $\mathrm{B}(q)$ at mid to low- $q$ and power law function, $\mathrm{C}(q)$ at very low- $q$ as given in equation 3 .

$$
I(q)=A(q)+B(q)+C(q) \ldots(3)
$$

The estimated structural parameters are given in Table 4. The Guinier-Porod model calculates scattering for a generalized Guinier/power law object that can be used to determine the size and dimensionality of scattering objects [71]. The DAB model calculates the scattering from a randomly distributed, two-phase system characterized by a single length scale, the correlation length (L), which is a measure of the average spacing between regions of the two phases. The model also assumes smooth interfaces between the phases and hence exhibits Porod behavior $q^{-4}$ at high- $q$ [72]. From USANS and SANS data fits (Fig. S4 in Supplementary Information), the average hydrophobic domain size in RSF hydrogel was estimated to be $\sim 2.3 \mathrm{~nm}$, which is randomly distributed in the amorphous matrix containing hydrophilic domains of silk fibroin with a correlation length of $\sim 4.8 \mathrm{~nm}$. A schematic representation of the proposed hydrogel structure is given in Fig. 5. 

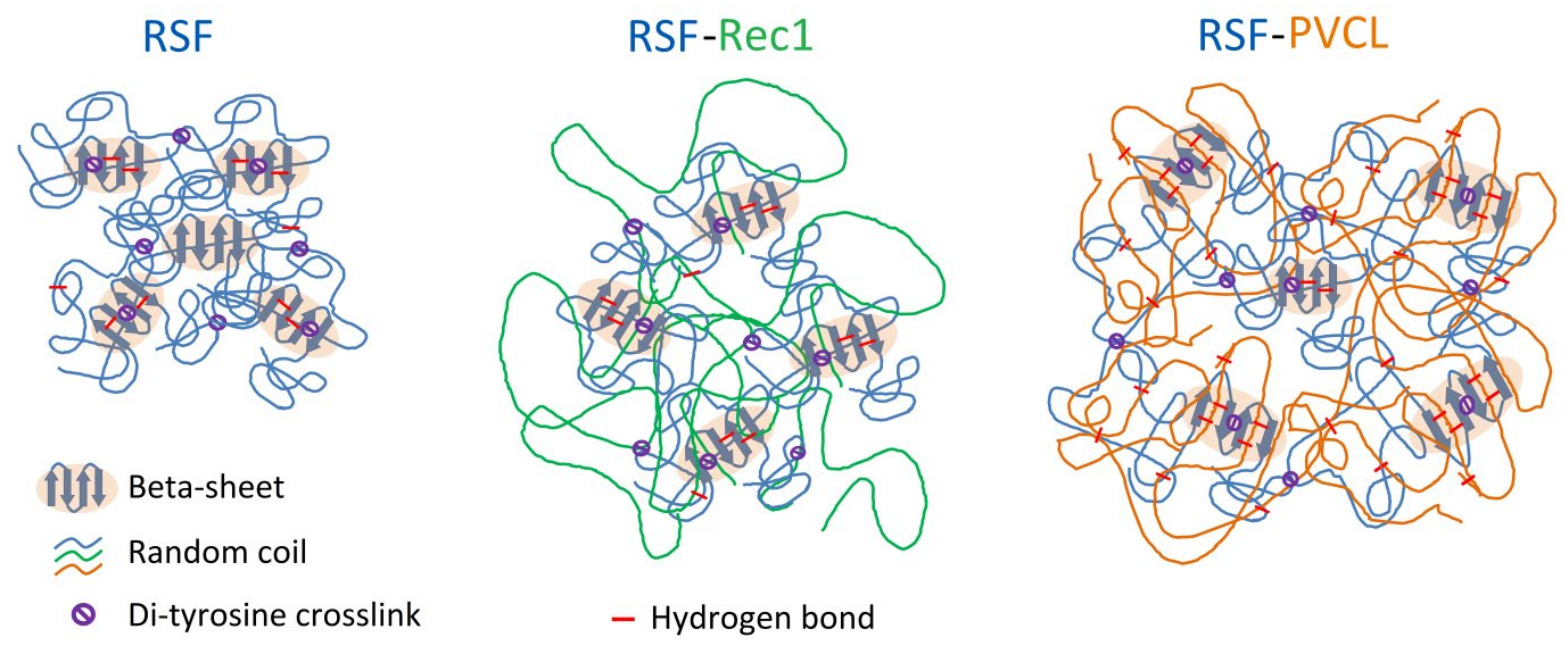

Fig. 5. A schematic of fabricated hydrogel structures in nanoscale. 
Table 4. Structural parameters of fabricated hydrogels determined from shape-independent model function fit to combined SANS and USANS data.

\begin{tabular}{|c|c|c|c|c|c|}
\hline \multirow[t]{2}{*}{ Samples } & \multirow{2}{*}{$\begin{array}{c}\text { Power law fit } \\
\text { (high-q) } \\
\text { Porod slope }\end{array}$} & \multicolumn{4}{|c|}{ Power law (very low-q) + DAB (mid to low-q) + Guinier-Porod fit (high-q) } \\
\hline & & $\begin{array}{c}\text { Low-q Porod } \\
\text { slope }\end{array}$ & Correlation length (nm) & $\mathbf{R}_{\mathbf{g}}(\mathbf{n m})$ & $\begin{array}{c}\text { High-q Porod } \\
\text { slope }\end{array}$ \\
\hline RSF100 gel & $4.00 \pm 0.01$ & $3.06 \pm 0.04$ & $4.78 \pm 0.36$ & $2.29 \pm 0.01$ & $4.00 \pm 0.01$ \\
\hline RSF50 Resilin50 gel & $3.54 \pm 0.02$ & $3.41 \pm 0.05$ & $5.07 \pm 0.51$ & $2.86 \pm 0.01$ & $3.54 \pm 0.03$ \\
\hline RSF90 PVCL10 gel & $3.86 \pm 0.02$ & $3.04 \pm 0.03$ & $6.05 \pm 0.55$ & $2.57 \pm 0.03$ & $3.86 \pm 0.03$ \\
\hline RSF80 PVCL20 gel & $3.72 \pm 0.02$ & $3.04 \pm 0.02$ & $9.13 \pm 0.81$ & $2.77 \pm 0.04$ & $3.72 \pm 0.03$ \\
\hline RSF70 PVCL30 gel & $3.60 \pm 0.03$ & $3.02 \pm 0.01$ & $15.07 \pm 1.16$ & $2.82 \pm 0.05$ & $3.60 \pm 0.05$ \\
\hline
\end{tabular}




\subsection{Supramolecular structure of photocrosslinked RSF-based hybrid hydrogels}

Fig. 6A compares the combined SANS and USANS curves (normalized) of equilibrium swollen RSF and RSF/Rec1-resilin hybrid hydrogel, whereas Fig. 6B compares RSF and RSF/PVCL hybrid hydrogels. The SANS curves of hybrid hydrogels clearly showed three distinct scattering regions similar to that of the RSF hydrogel. From the neutron scattering curve of RSF/Rec1-resilin hybrid hydrogel, the shoulder peak in USANS region was observed to be less pronounced, suggesting decrease in micropore structure regularity of RSF hydrogel with Rec-resilin substitution and photocrosslinking. A similar systematic trend was also observed for RSF/PVCL hybrid hydrogels. However, with 40\% PVCL substitution a well pronounced broad correlation peak was observed (discussed latter) in relatively higher $q$ value (lower size). On the other hand, the RSF/Rec1-resilin hybrid hydrogel showed a plateau or a slightly increased slope in Guinier region followed by a slight decrease in Porod region slope, suggesting size and/or structural changes in hydrophilic and hydrophobic domains of RSF hydrogel with Rec-resilin substitution. A systematic increase in Guinier slope and decrease in Porod slope was clearly observed for RSF/PVCL hybrid hydrogels with increase in PVCL substitution and photocrosslinking, suggesting changes in the hydrophilic and hydrophobic domain structures in RSF hydrogel. The Kratky plot of hybrid hydrogels (Fig. 6C and Fig. 6D) clearly showed a less pronounced USANS correlation peak, a highly pronounced mid- $q$ correlation peak and a well pronounced very high- $q$ scattering intensity. Interestingly, with increase in PVCL content, the mid- $q$ and high- $q$ correlation peak of RSF/PVCL hybrid hydrogel shifted to low- $q$, which suggests increase in domain size. Therefore, substitution of Rec1-resilin or PVCL and photocrosslinking changes the size and structure of micropores and hydrophilic/hydrophobic domains in RSF hydrogel. The structural parameters of hybrid hydrogels estimated from SANS data using the shape-independent form factor function (equation 3) is given in Table 4. 

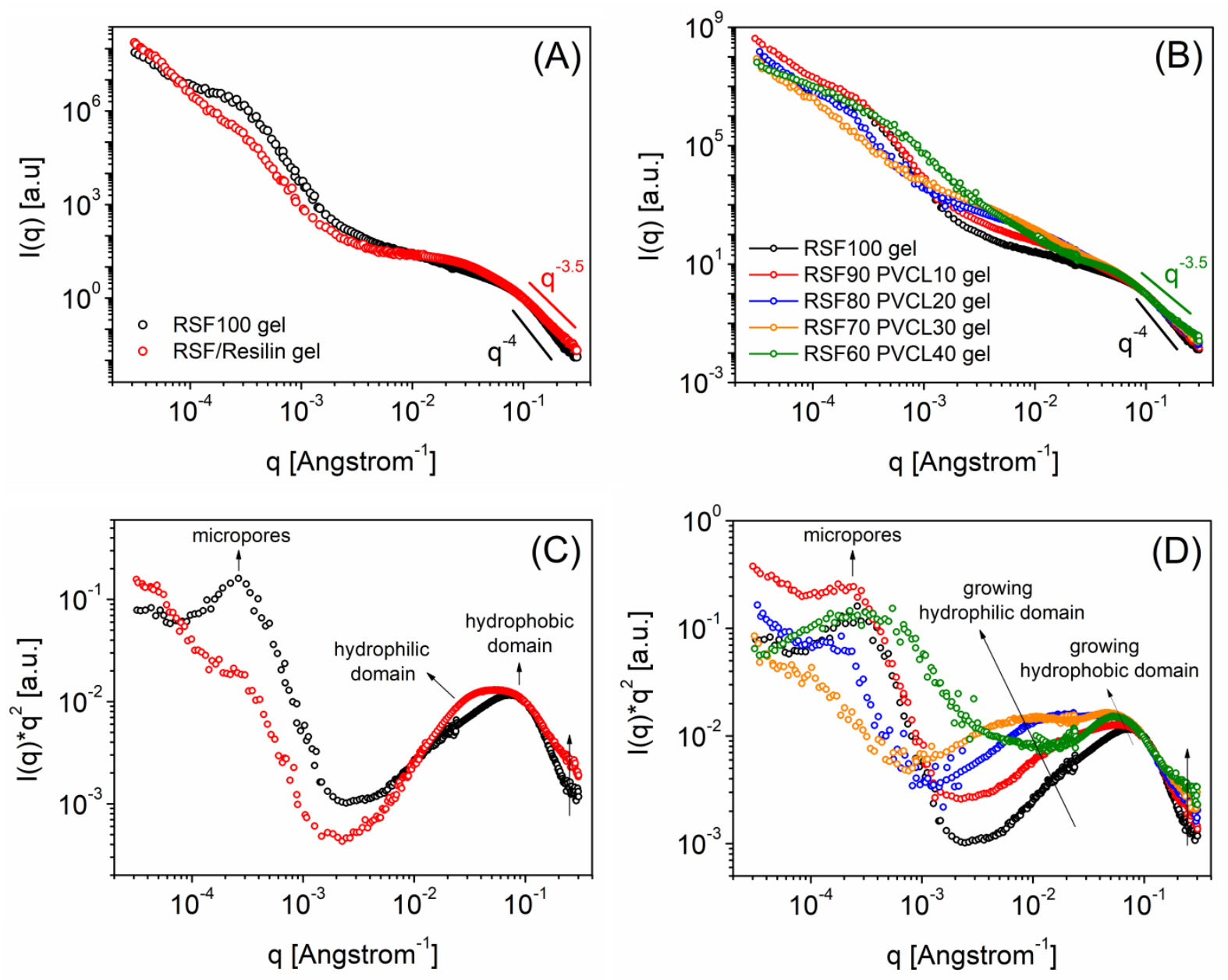

Fig. 6. Combined SANS and USANS curves (normalized) of (A) RSF/Rec1-resilin and (B) RSF/PVCL hybrid hydrogels. Kratky plots of (C) RSF/Rec1-resilin and (D) RSF/PVCL hybrid hydrogels.

The photocrosslinked hybrid hydrogels showed systematic decrease in high- $q$ Porod slope $(\sim 4$ to $\sim 3.5)$, whereas increase in both hydrophobic and hydrophilic domain size with increase in Rec1-resilin or PVCL substitution. Co-localization of tyrosine residues have been reported to increase the $\beta$-sheet size and content in silk fibroin-based materials [66]. However, crosslinking of tyrosine residues in RSF protein restricts the co-localization of tyrosine, thereby preventing increase in $\beta$-sheet size and content [73]. In our fabricated hydrogels, the RSF molecules are crosslinked by di-tyrosine covalent bonds formed between tyrosine molecules of proteins. Photocrosslinking RSF in the presence of Rec1-resilin or PVCL showed decreased 
crosslink density (higher molecular weight between crosslinks) in the hybrid hydrogel network (presented in our previous publication) $[16,17]$. However, this may not have influenced the restrictive co-localization of tyrosine residues in the hybrid hydrogels, where the hybrid hydrogels showed no noticeable change in secondary structure compared to RSF hydrogel (presented in our previous publication) $[16,17]$. Therefore, the change in overall high- $q$ Porod slope observed with increase in scattering intensity (showing power law behaviour of $q^{-2}$, Fig. 6C and Fig. 6D) can be attributed to the increased contribution from the uncollapsed Rec1resilin and/or PVCL chains, thereby increasing the hydrophobic domain size and decreasing its hydrophobicity (i.e. relatively low contrast). The systematic increase in hydrophilic domain size of hybrid hydrogels observed with Rec1-resilin or PVCL substitution and photocrosslinking is attributed to decrease in hydrogel crosslink density, thereby forming loose network structures favouring higher water uptake $[16,17]$. The increase in hydrophilic domain size of hybrid hydrogels with Rec1-resilin or PVCL substitution is also attributed to increase in amorphous nature of the hybrid hydrogels, which is observed with decrease in their glass transition temperature and increase in enthalpy of free water measured using differential scanning calorimetry (presented in our previous publication) $[16,17]$. Moreover, for hybrid hydrogel systems a single $T_{g}$ indicates miscible system with favourable interaction between components [16, 17]. During crosslinking reaction, the Rec1-resilin interacts with RSF through formation of both di-tyrosine crosslinks and hydrogen bonds, whereas PVCL interact with RSF only through hydrogen bonds, which thereby shows relatively lower crosslink density and higher correlation length $[16,17,23]$. On the other hand, the low- $q$ micropore correlation peak of RSF hydrogel moved slightly to higher- $q$ value (decrease in size) with Rec1-resilin, whereas to slightly lower- $q$ value (increase in size) with PVCL substitution and photocrosslinking. The low- $q$ correlation peak became less pronounced with higher Rec1-resilin or PVCL substitution clearly showing the $q^{-3}$ power law behaviour of hybrid hydrogels in USANS region. This 
behaviour is better visible in the Kratky plot. However, between $30 \%$ and $40 \%$ PVCL substitution there are structural changes that do not conform to the above trend. PVCL substitution of $40 \%$ resulted in a new peak (broad and intense) around $q$ value of $0.0006 \AA^{-1}$ corresponding to a domain size of $\sim 1 \mu \mathrm{m}$. The $40 \%$ PVCL sample was also observed to show a sharp and significant drop in crosslink density and mechanical modulus about two orders in magnitude (presented in our previous publication) [17]. Therefore, the low- $q$ correlation peak in $40 \%$ PVCL substituted hybrid hydrogel may be attributed to phase separated aggregates (poor network structure), where the RSF content in the hybrid hydrogel drops below $10 \mathrm{wt} \%$. Nevertheless, the inhomogeneity in network structure cannot be totally attributed to phase separation. Some structural defects in the network such as loops or dangling ends that get trapped into place as the gels are cross-linked can also give rise to such heterogeneity.

\section{Conclusions}

In summary, we have studied the structural evolution of RSF from dilute to concentrated solution to photo-chemically crosslinked hydrogel using small angle scattering techniques. The hierarchical structure of photocrosslinked pristine RSF and RSF-based hybrid hydrogels (RSF/Rec1-resilin and RSF/PVCL) were investigated over four orders of magnitude ( $\sim 1 \mathrm{~nm}$ to $20 \mu \mathrm{m}$ ) using SANS and USANS. The structural parameters of the fabricated hydrogels were elucidated using power law, DAB and Guinier-Porod model fits to SANS and USANS data. The RSF hydrogel exhibited crosslinked network structure with hydrophobic and hydrophilic domains in nanoscale and interconnected pores in the microscale. The RSF-based hybrid hydrogels showed increase in hydrophobic and hydrophilic domain sizes, whereas decrease in micropore size distribution and homogeneity with Rec1-resilin or PVCL substitution and photocrosslinking. Compared to RSF/Rec1-resilin hybrid hydrogel, the change in nanoscale domain sizes is relatively well pronounced in RSF/PVCL hybrid hydrogels. However, the observed trend breaks at 40\% PVCL substitution. This study provides insight into the RSF- 
based co-crosslinked and double network gel structures on the molecular level and elucidates the supramolecular structure over a range of length scales.

\section{Acknowledgements}

This research has been financially supported by the Australian Research Council (ARC) through Discovery Grant funding (DP160101267 and DP120103537). The SAXS, SANS and USANS experiments were supported by the ANSTO beam time award (P3550).

\section{Declaration of Competing Interest}

There are no conflicts of interest to declare

\section{References}

[1] A.M. Jonker, D.W.P.M. Löwik, J.C.M. van Hest, Chem. Mater., 24 (2012) 759-773.

[2] P. Alam, G. Rabbani, G. Badr, B.M. Badr, R.H. Khan, Cell Biochem. Biophys., 71 (2015) 1199-1206.

[3] A.S. Abdelhameed, P. Alam, R.H. Khan, J. Biomol. Struct. Dyn., 34 (2016) 2037-2044.

[4] B. Kundu, N.E. Kurland, S. Bano, C. Patra, F.B. Engel, V.K. Yadavalli, S.C. Kundu, Prog. Polym. Sci., 39 (2014) 251-267.

[5] A.E. Thurber, F.G. Omenetto, D.L. Kaplan, Biomaterials, 71 (2015) 145-157.

[6] R.D. Abbott, E.P. Kimmerling, D.M. Cairns, D.L. Kaplan, ACS Appl. Mater. Interfaces, 8 (2016) 21861-21868.

[7] E.M. Ahmed, J. Adv. Res., 6 (2015) 105-121.

[8] D.N. Rockwood, R.C. Preda, T. Yücel, X. Wang, M.L. Lovett, D.L. Kaplan, Nat. Protoc., 6 (2011) 1612.

[9] M. Floren, C. Migliaresi, A. Motta, J. Funct. Biomater., 7 (2016) 26.

[10] S. Kapoor, S.C. Kundu, Acta Biomater., 31 (2016) 17-32. 
[11] J.L. Whittaker, N.R. Choudhury, N.K. Dutta, A. Zannettino, J. Mater. Chem. B, 2 (2014) 6259-6270.

[12] C.G. Armstrong, V.C. Mow, J. Bone. Joint. Surg. Am., 64 (1982) 88-94.

[13] J.L. Whittaker, N.K. Dutta, R. Knott, G. McPhee, N.H. Voelcker, C. Elvin, A. Hill, N.R. Choudhury, Langmuir, 31 (2015) 8882-8891.

[14] R. Balu, J. Whittaker, N.K. Dutta, C.M. Elvin, N.R. Choudhury, J. Mater. Chem. B, 2 (2014) 5936-5947.

[15] N.A. Cortez-Lemus, A. Licea-Claverie, Progress Polym. Sci., 53 (2016) 1-51.

[16] J.L. Whittaker, N.K. Dutta, C.M. Elvin, N.R. Choudhury, J. Mater. Chem. B, 3 (2015) 6576-6579.

[17] J.L. Whittaker, N.K. Dutta, A. Zannettino, N.R. Choudhury, J. Mater. Chem. B, 4 (2016) 5519-5533.

[18] Q. Wang, Q. Chen, Y. Yang, Z. Shao, Biomacromolecules, 14 (2013) 285-289.

[19] H. Yamada, H. Nakao, Y. Takasu, K. Tsubouchi, Mater. Sci. Eng. C, 14 (2001) 41-46.

[20] H. Zhang, L.-1. Li, F.-y. Dai, H.-h. Zhang, B. Ni, W. Zhou, X. Yang, Y.-z. Wu, J. Transl. Med., 10 (2012) 117.

[21] Z. Zheng, S. Guo, Y. Liu, J. Wu, G. Li, M. Liu, X. Wang, D. Kaplan, J. Biomater. Appl., 31 (2016) 450-463.

[22] S. Nagarkar, T. Nicolai, C. Chassenieux, A. Lele, Phys. Chem. Chem. Phys., 12 (2010) 3834-3844.

[23] Zainuddin, T.T. Le, Y. Park, T.V. Chirila, P.J. Halley, A.K. Whittaker, Biomaterials, 29 (2008) 4268-4274.

[24] A. Martel, M. Burghammer, R.J. Davies, E. Di Cola, C. Vendrely, C. Riekel, J. Am. Chem. Soc., 130 (2008) 17070-17074. 
[25] I. Greving, C. Dicko, A. Terry, P. Callow, F. Vollrath, Soft Matter, 6 (2010) 43894395.

[26] J. Zhong, X. Liu, D. Wei, J. Yan, P. Wang, G. Sun, D. He, Int. J. Biol. Macromol., 76 (2015) 195-202.

[27] Q. Lu, H. Zhu, C. Zhang, F. Zhang, B. Zhang, D.L. Kaplan, Biomacromolecules, 13 (2012) 826-832.

[28] H.D.T. Mertens, D.I. Svergun, J. Struct. Biol., 172 (2010) 128-141.

[29] R. Balu, R. Knott, N.P. Cowieson, C.M. Elvin, A.J. Hill, N.R. Choudhury, N.K. Dutta, Sci. Rep., 5 (2015) 10896.

[30] M. Shibayama, Polym. J., 43 (2011) 18-34.

[31] R. Balu, J.P. Mata, R. Knott, C.M. Elvin, A.J. Hill, N.R. Choudhury, N.K. Dutta, J. Phys. Chem. B, 120 (2016) 6490-6503.

[32] D.W. Schaefer, M.M. Agamalian, Curr. Opin. Solid State Mater. Sci., 8 (2004) 39-47.

[33] C.M. Elvin, A.G. Carr, M.G. Huson, J.M. Maxwell, R.D. Pearson, T. Vuocolo, N.E. Liyou, D.C.C. Wong, D.J. Merritt, N.E. Dixon, Nature, 437 (2005) 999.

[34] J. Lipfert, S. Doniach, Annu. Rev. Biophys. Biomol. Struct., 36 (2007) 307-327.

[35] P.V. Konarev, V.V. Volkov, A.V. Sokolova, M.H.J. Koch, D.I. Svergun, J. Appl. Crystallogr., 36 (2003) 1277-1282.

[36] E.P. Gilbert, J.C. Schulz, T.J. Noakes, Physica B: Condensed Matter., 385-386, Part 2 (2006) 1180-1182.

[37] D. Jayawardane, F. Pan, J.R. Lu, X. Zhao, Langmuir, 32 (2016) 8202-8211.

[38] F. Schneider, A. Balaceanu, A. Feoktystov, V. Pipich, Y. Wu, J. Allgaier, W. Pyckhout-Hintzen, A. Pich, G.J. Schneider, Langmuir, 30 (2014) 15317-15326.

[39] S. Kline, J. Appl. Crystallogr., 39 (2006) 895-900. 
[40] C. Rehm, A. Brule, A.K. Freund, S.J. Kennedy, J. Appl. Crystallogr., 46 (2013) 16991704.

[41] T. Lam, N. Hauser, A. Götz, P. Hathaway, F. Franceschini, H. Rayner, L. Zhang, Physica B: Condensed Matter., 385 (2006) 1330-1332.

[42] E.M. Saffer, M.A. Lackey, D.M. Griffin, S. Kishore, G.N. Tew, S.R. Bhatia, Soft Matter, 10 (2014) 1905-1916.

[43] S. Lin, G. Lu, S. Liu, S. Bai, X. Liu, Q. Lu, B. Zuo, D.L. Kaplan, H. Zhu, J. Mater. Chem. B, 2 (2014) 2622-2633.

[44] C.D. Putnam, M. Hammel, G.L. Hura, J.A. Tainer, Quarter. Rev. Biophys., 40 (2007) 191-285.

[45] O. Glatter, O. Kratky, Small Angle X-ray Scattering, Academic Press, 1982.

[46] D.I. Svergun, M.H.J. Koch, P.A. Timmins, R.P. May, Small Angle X-Ray and Neutron Scattering from Solutions of Biological Macromolecules, OUP Oxford, 2013.

[47] R.-B. Veronique, D. Dominique, Curr. Protein Pept. Sci., 13 (2012) 55-75.

[48] H.J. Cho, C.S. Ki, H. Oh, K.H. Lee, I.C. Um, Int. J. Biol. Macromol., 51 (2012) 336341.

[49] P. Bernadó, Eur. Biophys. J., 39 (2010) 769-780.

[50] A.C.W. Lau, C. Wu, Macromolecules, 32 (1999) 581-584.

[51] R.J. Ellis, A.P. Minton, Biol. Chem., 387 (2006), 485-497.

[52] Y. Zhang, C. Zhang, L. Liu, D.L. Kaplan, H. Zhu, Q. Lu, Front. Mater. Sci., 9 (2015) $382-391$

[53] J.L. Whittaker, Engineered silk-based biomimetic hydrogels for cell growth, $\mathrm{PhD}$ thesis, University of South Australia, 2016.

[54] J.M. Ruso, Á. Piñeiro, Proteins in Solution and at Interfaces: Methods and Applications in Biotechnology and Materials Science, Wiley, 2013. 
[55] L. Dai, Intelligent Macromolecules for Smart Devices: From Materials Synthesis to Device Applications, Springer London, 2006.

[56] S.H. Chen, Annu. Rev. Phys. Chem., 37 (1986) 351-399.

[57] C. De, J. Appl. Crystallogr., 47 (2014) 1479-1489.

[58] N. Drnovsek, R. Kocen, A. Gantar, M. Drobnic-Kosorok, A. Leonardi, I. Krizaj, A. Recnik, S. Novak, J. Mater. Chem. B, 4 (2016) 6597-6608.

[59] F. Horkay, P.J. Basser, A.-M. Hecht, E. Geissler, Polymer, 46 (2005) 4242-4247.

[60] M.A. Iannuzzi, R. Reber, D.M. Lentz, J. Zhao, L. Ma, R.C. Hedden, Polymer, 51 (2010) 2049-2056.

[61] S. Kundu, Silk Biomaterials for Tissue Engineering and Regenerative Medicine, Elsevier Science, 2014.

[62] S.-W. Ha, H.S. Gracz, A.E. Tonelli, S.M. Hudson, Biomacromolecules, 6 (2005) 25632569.

[63] M.A. Koperska, D. Pawcenis, J. Bagniuk, M.M. Zaitz, M. Missori, T. Łojewski, J. Łojewska, Polym. Degrad. Stab., 105 (2014) 185-196.

[64] M. Mondal, Casp. J. Env. Sci., 5 (2007) 63-76.

[65] T. Asakura, R. Sugino, J. Yao, H. Takashima, R. Kishore, Biochemistry, 41 (2002) $4415-4424$.

[66] B.P. Partlow, M. Bagheri, J.L. Harden, D.L. Kaplan, Biomacromolecules, 17 (2016) $3570-3579$.

[67] S. Kalia, Polymeric Hydrogels as Smart Biomaterials, Springer International Publishing, 2015.

[68] H.D. Bale, P.W. Schmidt, Phys. Rev. Lett., 53 (1984) 596-599.

[69] L.A. Feigin, D.I. Svergun, Structure Analysis by Small-Angle X-Ray and Neutron Scattering, Springer US, 2013. 
[70] A. Radulescu, G. Goerigk, L. Fetters, D. Richter, J. Appl. Crystallogr., 48 (2015) 18601869.

[71] B. Hammouda, J. Appl. Crystallogr., 43 (2010) 716-719.

[72] P. Debye, H.R.A. Jr., H. Brumberger, J. Appl. Phys., 28 (1957) 679-683.

[73] D. Su, M. Yao, J. Liu, Y. Zhong, X. Chen, Z. Shao, ACS Appl. Mater. Interfaces, 9 (2017) 17489-17498. 


\section{Supporting Information}

\section{S1. Preparation and purification of regenerated silk fibroin (RSF)}

The raw silk of $B$. mori was first degummed (removal of sericin protein) by boiling them in aqueous $0.02 \mathrm{M}$ sodium carbonate for $30 \mathrm{~min}$. The boiled silk was rinsed with distilled water three times and allowed to dry at room temperature overnight. The dried silk was dissolved in a calcium chloride-water-ethanol solution mixture $\left(1: 8: 2\right.$ mole ratio) at $70{ }^{\circ} \mathrm{C}$ for 3 hour with a liquor ratio of 1:10. The dissolved silk solution was dialysed against MilliQ water for 5 days (with fresh water changed daily) using 3,500 MWCO SnakeSkin $^{\mathrm{TM}}$ dialysis tube (ThermoFisher). The dialysed solution was centrifuged at $10,000 \mathrm{rpm}$ for $30 \mathrm{~min}$. The obtained supernatant was filtered using a 1.2 micron syringe filter (Sartorius). The filtered supernatant was concentrated by air-drying at ambient temperature and refiltered [1]. The concentration of obtained RSF solution was determined to be approximately $150 \mathrm{mg} / \mathrm{mL}$. The obtained RSF solution was stored at $4{ }^{\circ} \mathrm{C}$ to prevent gelation and used within three days.

\section{S2. Synthesis and purification of Rec1-resilin}

Briefly, the exon-1 of D. melanogaster CG15920 gene was first cloned and expressed as a water soluble protein in the bacteria $E$. coli using a lactose-induced fermentation system, as reported elsewhere [2]. The obtained protein was then purified and concentrated by a salt precipitation method using $20 \%$ ammonium sulphate, followed by overnight dialysis in excess phosphate-buffered saline at $4{ }^{\circ} \mathrm{C}$ and heating at $80{ }^{\circ} \mathrm{C}$ for 10 min under stirring. The denatured proteins were then removed by centrifugation at $12,000 \mathrm{~g}$ for $15 \mathrm{~min}$ and the resulting protein solution in supernatant was stored in freezer. [3].

\section{S3. Synthesis and purification of Poly(N-vinylcaprolactam) (PVCL)}

PVCL was prepared via free radical polymerisation of the vinylcaprolactam monomer with 2,2'-azoisobutyronitrile (AIBN) as the initiator and toluene as the solvent. The polymerisation 
reaction proceeded for 24 hour under nitrogen atmosphere at $70{ }^{\circ} \mathrm{C}$. The synthesized polymer was then recovered through precipitation in diethyl ether. The obtained PVCL was purified by dissolving it in a mixture of acetone and water $(3: 7 \mathrm{v} / \mathrm{v})$, followed by precipitation by heating at $80{ }^{\circ} \mathrm{C}$ [4]. This process was repeated twice, and the purified PVCL was dried at room temperature, followed by vacuum drying at $50{ }^{\circ} \mathrm{C}$ for 3 hour to remove any residual moisture.

\section{S4. Molecular weight determination of synthesized macromolecules}

The molecular weight of RSF was determined using sodium dodecyl sulfate-polyacrylamide gel electrophoresis (SDS-PAGE). Briefly, about $20 \mu \mathrm{g}$ of RSF in $7.5 \mu \mathrm{L}$ of MilliQ water was mixed with $2.5 \mu \mathrm{L}$ of NuPAGE ${ }^{\circledR} 4 \mathrm{x}$ LDS sample buffer. The prepared sample was loaded into a SDS-PAGE electrophoresis system made from NuPAGE ${ }^{\circledR}$ Novex $^{\circledR} 4-12 \%$ Bis-Tris Protein Gel and MES SDS 1x running buffer. The protein marker was loaded into the first lane (left) and the protein into the second lane. An electric potential of 140 volts was applied to the SDSPAGE electrophoresis system for about 1.5 hour. The gel was then stained using Coomassie brilliant blue for 1 hour (incubated on a rocking table) and gently rinsed twice with double distilled water. Destaining was performed by incubation of the gel in destain solution for 1 hour. The gel was then gently rinsed with double distilled water and observed under UV light [5]. Compared with standard marker, the apparent molecular weight of RSF was determined to be approximately 37 to $200 \mathrm{kDa}$ by mobility of protein in the gel (Fig. S1).

The molecular weight of synthesized Rec1-resilin was determination by matrix assisted laser desorption ionization-time of flight mass spectrometry (MALDI-TOF MS). Briefly, pure protein $(4.8 \mathrm{mg}$ ) was dissolved in $30 \%$ acetonitrile, $0.1 \%$ trifluoroacetic acid (TA30) to obtain a concentration of $3.0 \mathrm{mg} / \mathrm{ml}$. About $1.5 \mu \mathrm{l}$ of matrix (sinapinic acid, saturated in ethanol) was pre-spotted onto a polished steel target plate (Bruker Daltonics, Germany) and air dried. Now, $2 \mu \mathrm{l}$ of protein solution $(1 \mathrm{pmol} / \mu \mathrm{l}$ ) was mixed with $2 \mu \mathrm{l}$ matrix (sinapinic acid, saturated in TA30), and $0.5 \mu 1$ of sample-matrix mix was spotted onto the previously created matrix spots 
and air dried. Mass spectra were acquired on an ultrafleXtreme MALDI-TOF mass spectrometer (Bruker Daltonics) operating in linear positive mode. Instrument settings were set in flexControl software (Version 3.4, Bruker Daltonics). Measurement range was set to $\mathrm{m} / \mathrm{z}$ $5000-50,000.5000$ shots were collected for the external calibration and 20,000 shots for sample measurement. External calibration was performed using a mix of protein calibration standard I and II (Bruker Daltonics). Laser intensity and detector gain were manually adjusted for optimal resolution. The MS spectra obtained (Fig. S2) was analysed using flexAnalysis software (version 3.3, Bruker Daltonics) employing smoothing, background subtraction and peak detection algorithms. The molecular weight of synthesised Rec1-resilin was determined to be $\sim 28.5 \mathrm{kDa}$.

The viscosity average molecular weight $\left(\mathrm{M}_{\mathrm{v}}\right)$ of the synthesised PVCL was determined from the intrinsic viscosity ([ๆ]) by employing the Mark-Houwink equation:[6]

$$
[\eta]=\mathrm{K}^{*} \mathrm{M}_{\mathrm{v}}{ }^{\mathrm{a}} \ldots(1)
$$

where $K=0.0105 \mathrm{ml} / \mathrm{g}$ and $a=0.69$ [6]. An Ubbelohde-type viscometer was used with water as the solvent. The reduced viscosity $\left(\eta_{\text {red }}\right)$ and inherent viscosity $\left(\eta_{\text {inh }}\right)$ at $25{ }^{\circ} \mathrm{C}$ were

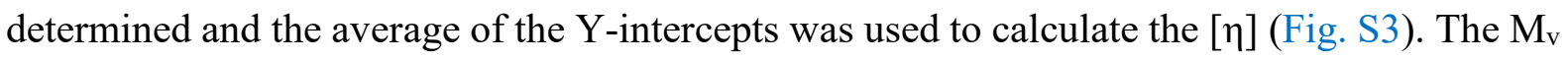
of synthesised PVCL was determined to be $\sim 35.4 \mathrm{kDa}$. 


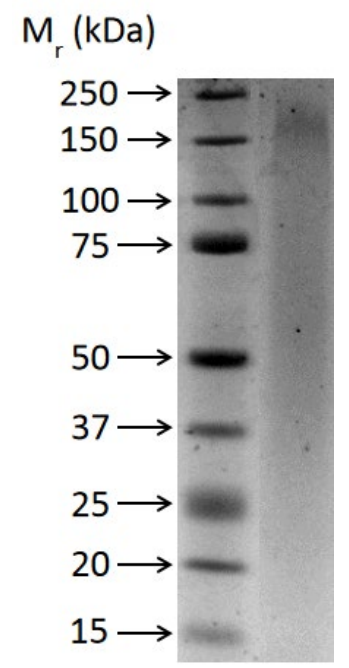

Fig. S1. SDS-PAGE picture of RSF (right lane).

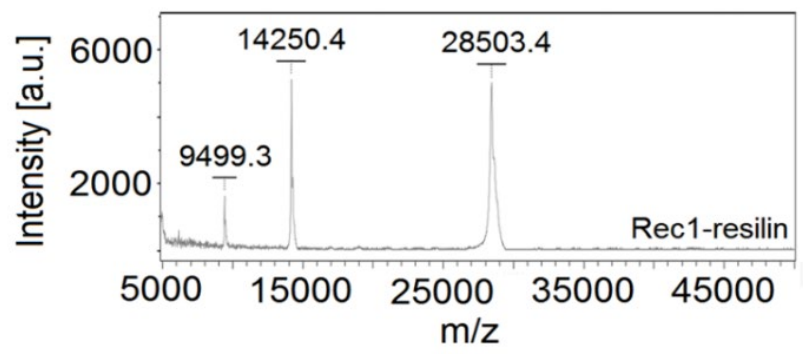

Fig. S2. MALDI-TOF mass spectra of synthesized Rec1-resilin. The three $\mathrm{m} / \mathrm{z}$ species (right to left) detected in mass spectra are the $[\mathrm{M}+\mathrm{H}]^{+},[\mathrm{M}+2 \mathrm{H}]^{2+}$, and $[\mathrm{M}+4 \mathrm{H}]^{4+}$ species. 


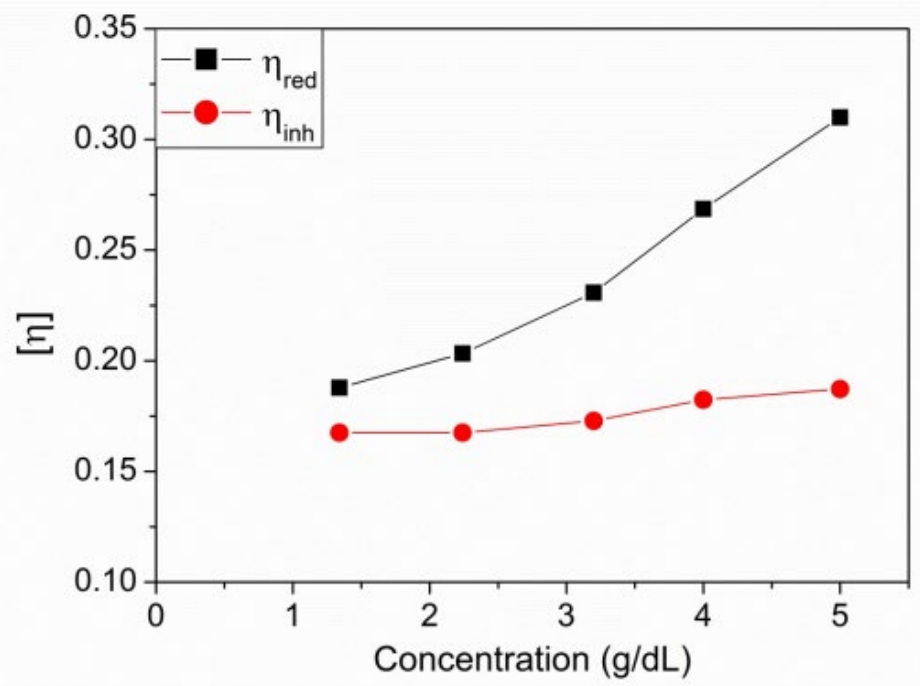

Fig. S3. Plot of reduced and inherent viscosities determined for PVCL. 

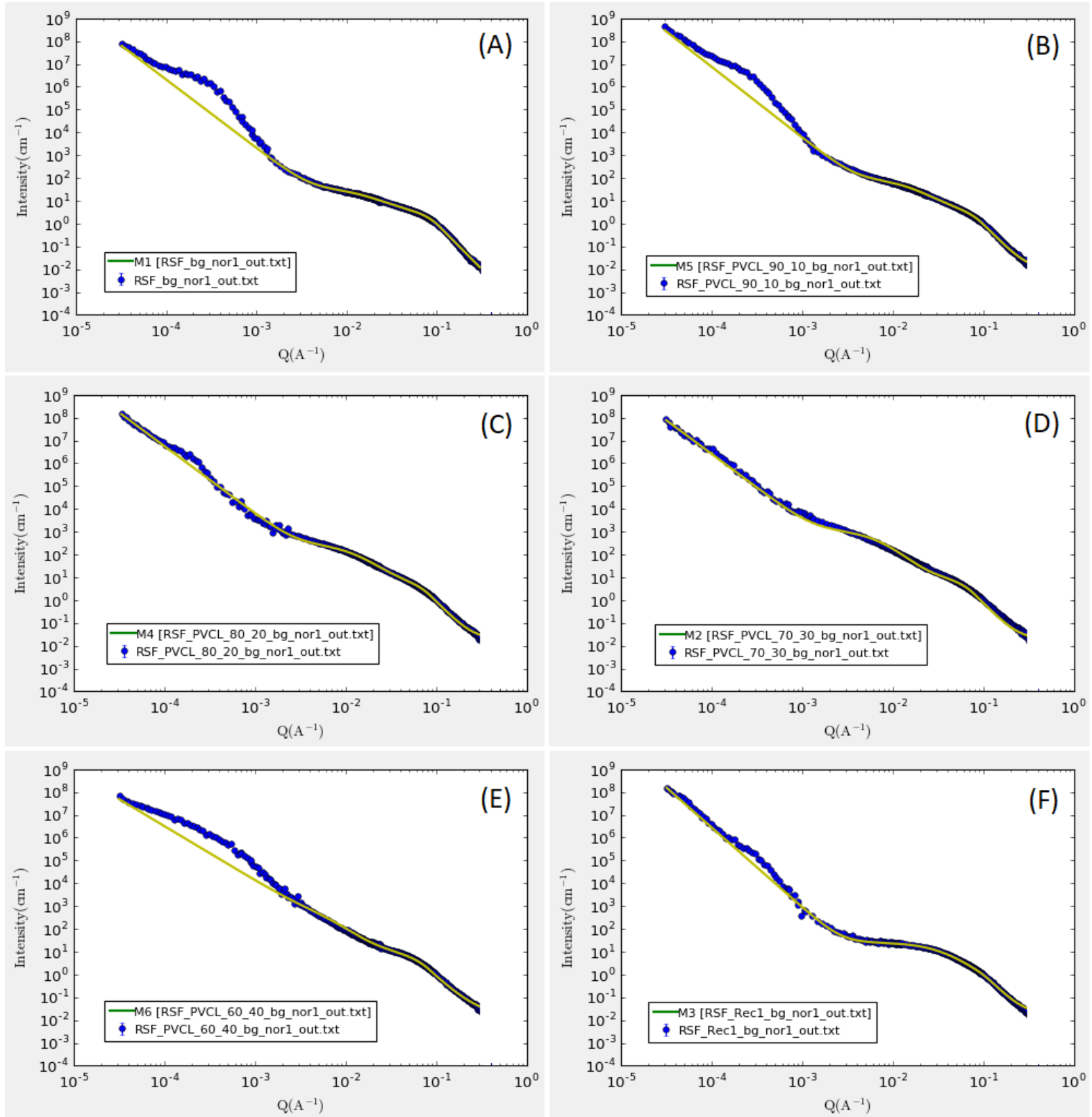

Fig. S4. Combined shape-independent form factor function (Power law + DAB + GuinierPorod) fits to USANS and SANS data of (A) RSF100 gel, (B) RSF90 PVCL10 gel, (C) RSF80 PVCL20 gel, (D) RSF70 PVCL30 gel, (E) RSF60 PVCL40 gel and (F) RSF50 Resilin50 gel. 


\section{References:}

[1] J.L. Whittaker, N.R. Choudhury, N.K. Dutta, A. Zannettino, J. Mater. Chem. B, 2 (2014) $6259-6270$.

[2] M. Kim, C. Elvin, A. Brownlee, R. Lyons, Protein Expression Purif., 52 (2007) 230-236.

[3] R.E. Lyons, E. Lesieur, M. Kim, D.C.C. Wong, M.G. Huson, K.M. Nairn, A.G. Brownlee, R.D. Pearson, C.M. Elvin, Protein Eng., Design Select., 20 (2007) 25-32.

[4] J.L. Whittaker, N.K. Dutta, A. Zannettino, N.R. Choudhury, J. Mater. Chem. B, 4 (2016) $5519-5533$.

[5] J.L. Whittaker, Engineered silk-based biomimetic hydrogels for cell growth, $\mathrm{PhD}$ thesis, University of South Australia, 2016.

[6] W. Loos, S. Verbrugghe, E.J. Goethals, F.E. Du Prez, I.V. Bakeeva, V.P. Zubov, Macromol. Chem. Phys., 204 (2003) 98-103. 